\title{
Formation Control of Multi-Robot using Virtual Structures with a Linear Algebra Approach
}

\author{
Arfittariah \\ Department of Electrical Enginering \\ Institut Teknologi Sepuluh Nopember \\ Surabaya, Indonesia \\ fittarhia@gmail.com
}

\author{
Trihastuti Agustinah \\ Department of Electrical Enginering \\ Institut Teknologi Sepuluh Nopember \\ Surabaya, Indonesia \\ trihastuti@ee.its.ac.id
}

\begin{abstract}
The separation bearing controller (SBC) and separation-separation controller (SSC) approach can be used to maintain multi-robot formation, while the Computed Torque Control (CTC) method is used to solve the dynamic problems of the robot. However, the SBC and SSC approach combined with CTC cannot maintain multi-robot formation, if the leader or follower is disturbed. In this paper, the virtual structure method and the linear algebra approach added to the CTC method are used to maintain the triangular robot formation to follow the given trajectory. The simulation results show that the controller that has increased the highest MSE value is, $\mathrm{MSE}_{x}=4.5152 \times 10^{-10}$ and $\mathrm{MSE} e_{y}=3.3945 \times 10^{-10}$, and the lowest $\mathrm{MSE} e_{x}=0.000010$ and MSE $e_{y}=0.000011$. For time trajectory on a circle with a value of less than $20 \mathrm{~s}$ and a number 8 with a value of 1 minute. But from the results of the overall robot can maintain the DDMR compilation formation doing trajectories in the form of a circle and number 8 , both without interference or in the effect of interference and noise.
\end{abstract}

Keywords-Computed Torque Control, Virtual Structure, Linear Algebra, Trajectory Tracking, Formation Control

\section{INTRODUCTION}

Differential Drive Mobile Robot (DDMR) is a type of mobile robot possessing two separately moving wheels, requiring adjustments to each wheel in order to move properly. An application of DDMR is in robot formation, where multiple robots move together in a formation. This usage type has already been used in areas such as shipping goods [1], search-and-rescue operations [2], and monitoring hazardous areas [3].

Robot formation requires arrangements for the movement of each robot to run on a trajectory by maintaining the desired formation form. The formation control can use the leader-follower approach where there is a robot leader to determine the path to be followed while the follower robot follows the robot leader by maintaining the desired formation. The leader-follower approach is found in the separation-bearing controller (SBC) and separationseparation controller (SSC). In SBC robot follower follows one robot leader by maintaining distance and angle of the bearing. Whereas SSC is maintaining the distance and angular bearing of the follower robot following other followers. These two controller schemes can be combined with computed torque control, or CTC, to control the dynamics of the robots in a nonlinear fashion. This scheme allows the 4-robot formation to traverse the given trajectory while maintaining the formation [4]. However, if a disturbance is introduced to the robot follower, they will not be able to maintain formation, even if they are equipped with a nonlinear disturbance observer (NDO) method for estimating disturbance signals. This is because there is no feedback from the robot follower to the leader.

Control of robot formation with feedback between leader and follower or vice versa can use a virtual structure-based method which uses a spring-damper approach on a triangular robot formation [5]. The advantage of the virtual structure method is that it can maintain triangular formations despite disturbance. But there is no coordination between robots in formation.

The robot formation control with a coordination system between robots can use a linear algebraic approach to calculate the control actions so that the robot formation reaches a predetermined trajectory [6]. The formation control uses a linear algebraic approach with two control variables namely, the linear and angular velocity of each robot.

From the previous problems and solutions, we then propose a formation control system to maintain a triangular formation while traversing a given trajectory.

This paper is organized as follows: Section II explains the derived kinematic and dynamic models of the mobile robot. The proposed method is explained in Section III. The simulations result and analysis of the proposed method are presented in section IV. Section V discusses the conclusion of this paper.

\section{KinEMATIC AND Dynamic Models IN Differential DRIVE MOBILE ROBOTS}

\section{A. Kinematic Model}

The kinematic and dynamic model of DDMR in the twodimensional coordinates $(x, y)$ is explained in this section. The DDMR kinematic model is needed to analyze the movement of the robot in the two dimensions. The linear velocity of a given DDMR is as follows:

$$
v=\frac{v_{r}+v_{l}}{2}=r \frac{\left(\dot{\varphi}_{r}+\dot{\varphi}_{l}\right)}{2}
$$

Whereas its angular velocity is as follows:

$$
\omega=\frac{v_{r}-v_{l}}{2 L}=r \frac{\left(\dot{\varphi}_{r}-\dot{\varphi}_{l}\right)}{2}
$$

Where $v_{r}$ and $v_{l}$ are the linear velocities of the left and right wheels, $r$ is the radius of the wheels. $L$ is the distance 
between the center point of the wheel to the centerline of the robot body.

Equations (1) and (2) may be represented in the velocity center point of the robot $\left(\dot{x}_{a}, \dot{y}_{a}\right.$ and $\left.\dot{\theta}\right)$ shown in Equation (3).

$$
\left\{\begin{array}{c}
\dot{x}_{a}=r \frac{\left(\dot{\varphi}_{r}+\dot{\varphi}_{l}\right)}{2} \\
\dot{y}_{a}=0 \\
\dot{\theta}=r \frac{\left(\dot{\varphi}_{r}-\dot{\varphi}_{l}\right)}{2}
\end{array}\right.
$$

Equation (3) may then be represented in a matrix multiplication as follows:

$$
\left[\begin{array}{c}
\dot{x}_{a} \\
\dot{y}_{a} \\
\dot{\theta}
\end{array}\right]=\left[\begin{array}{cc}
\frac{r}{2} & \frac{r}{2} \\
0 & 0 \\
\frac{r}{2 L} & \frac{r}{2 L}
\end{array}\right]\left[\begin{array}{l}
\dot{\varphi}_{r} \\
\dot{\varphi}_{l}
\end{array}\right]
$$

We obtain the kinematic DDMR equation in the inertial frame as follows:

$$
\left[\begin{array}{c}
\dot{x}_{a} \\
\dot{y}_{a} \\
\dot{\theta}
\end{array}\right]=\left[\begin{array}{ll}
\frac{r}{2} \cos \theta & \frac{r}{2} \cos \theta \\
\frac{r}{2} \sin \theta & \frac{r}{2} \sin \theta \\
\frac{r}{2 L} & -\frac{r}{2 L}
\end{array}\right]\left[\begin{array}{c}
\dot{\varphi}_{r} \\
\dot{\varphi}_{l}
\end{array}\right]
$$

This may also be represented as follows:

$$
\left[\begin{array}{c}
\dot{x}_{a} \\
\dot{y}_{a} \\
\dot{\theta}
\end{array}\right]=\left[\begin{array}{cc}
\cos \theta & 0 \\
\sin \theta & 0 \\
0 & 1
\end{array}\right]\left[\begin{array}{c}
\dot{\varphi}_{r} \\
\dot{\varphi}_{l}
\end{array}\right]
$$

\section{B. Dynamic Model}

The dynamic model is obtained by deriving the model using a Lagrange formula. The system's dynamic model is represented by the following equation [1]:

$$
M(q) \ddot{\eta}+V(q, \dot{q}) \dot{\eta}=B(q) \tau-A^{T}(q) \lambda
$$

where $M(q) \in \mathfrak{R}^{2 \times 2}$ is the positive definitive symmetric inertial matrix, $V(q, \dot{q}) \dot{\eta} \in \mathfrak{R}^{2 \times 2}$ is the centripetal and Coriolis matrices, $B(q) \in \mathfrak{R}^{2 \times 2}$ is the input matrix, $\tau \in \mathfrak{R}^{2 \times 1}$ is the torsion, $A^{T}(q) \in \mathfrak{R}^{2 \times 2}$ is matrix constraint and $\lambda \in \mathfrak{R}^{1 x 1}$ is the Lagrange multiplier.

This equation is very important in controlling the stability analysis of the robot system. Next is eliminating $A^{T}(q)$ in Equation (8) due to the unknown Lagrange modifier $\lambda$. Thus, the DDMR dynamic model is represented as follows:

$$
\bar{M}(q) \dot{\eta}+\bar{V}(q, \dot{q}) \eta=\bar{B}(q) \tau
$$

where,

$$
\bar{M}(q)=\left[\begin{array}{cc}
I_{w}+\frac{r^{2}}{4 b^{2}}\left(m L^{2}+I\right) & \frac{r^{2}}{4 b^{2}}\left(m L^{2}-I\right) \\
\frac{r^{2}}{4 b^{2}}\left(m L^{2}-I\right) & I_{w}+\frac{r^{2}}{4 b^{2}}\left(m L^{2}+I\right)
\end{array}\right]
$$

$\bar{V}(q, \dot{q})=\left[\begin{array}{cc}0 & \frac{r^{2}}{2 b} m_{c} d \dot{\theta} \\ -\frac{r^{2}}{2 b} m_{c} d \dot{\theta} & 0\end{array}\right]$

$\bar{B}(q)=\left[\begin{array}{ll}1 & 0 \\ 0 & 1\end{array}\right]$

$r=\left[\begin{array}{c}\tau_{r} \\ \tau_{l}\end{array}\right]$, which $m=m_{c}+2 m_{w}, I=I_{c}+m_{c} d^{2}+2 m_{w} L^{2}+2 I_{m}$.

\section{FORMATION CONTROL DESIGN}

Our research design controls robot formation in trajectory tracking using the linear algebra approach [6]. The designed formation control is based on the formation state concept and nonholonomic robot model dynamics. Using the formation state concept, the constituent robots may change their formation configuration in both size and shape to be able to properly follow the given trajectory.

\section{A. Kinematic Model Using Linear Algebra}

We use a first-order kinematic model, shown in the equation as follows:

$$
\dot{\xi}=\dot{\eta}
$$

where,

$\dot{\eta} \in \mathfrak{R}^{1 x 6}=\left[\begin{array}{llllll}\dot{x}_{1} & \dot{y}_{1} & \dot{x}_{2} & \dot{y}_{2} & \dot{x}_{3} & \dot{y}_{3}\end{array}\right]^{T}$

$\xi \in \mathfrak{R}^{1 x 6}=$ trivial vector in the formation space

The chosen formation is shown in Figure 1, wherein a triangular formation the main controlled variable is the robots' position towards their leader.

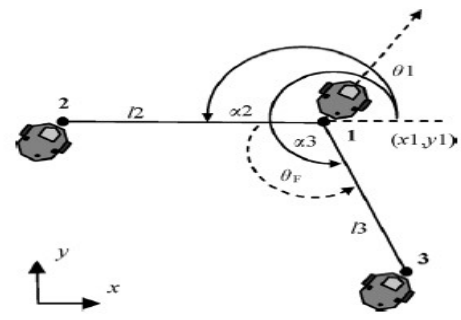

Fig. 7. Formation scheme for three robots [5]

\section{B. Formation Control Using Linear Algebra}

With the knowledge of the desired state, we may now calculate the needed control adjustments to track the reference trajectory. The reference velocity is available from the formation control, allowing the multi-robot system to reach the desired formation state with minimum error. The formation's kinematic model may be represented in Equation (11) as follows: 
JAREE-Journal on Advance Research in Electrical Engineering Volume4, Number 1, April 2020

$$
\left[\begin{array}{l}
\dot{x}_{1} \\
\dot{y}_{1}
\end{array}\right]=\left[\begin{array}{l}
k_{1 x}\left\{x_{1_{d}}-x_{1}\right\} \\
k_{1 y}\left\{y_{1_{d}}-y_{1}\right\}
\end{array}\right]
$$

where $\dot{\eta} \in \mathfrak{R}^{2 x 1}$ is velocity, $k_{1 x}$ and $k_{1 y} \in \mathfrak{R}^{2 x 1}$ are the positive constants, $x_{1_{d}}$ and $y_{1_{d}} \in \mathfrak{R}^{2 \times 1}$ are the desired position, while $x_{1}$ and $y_{1} \in \mathfrak{R}^{2 x 1}$ are the actual position.

\section{Computed Torque Control Design with Virtual Structure}

In our research, we designed a computed torque control, or CTC, as the dynamics control of a DDMR set. CTC is a control method applying feedback linearization to a nonlinear system [8]. The CTC equation in DDMR is shown in Equation (12) as follows:

$$
\tau=\bar{\beta}(q)^{-1}\left(\bar{M}(q)\left[\dot{\eta}+k_{p} e+k_{d} \dot{e}\right]+\bar{V}(q, \dot{q}) \eta\right)
$$

The CTC design with virtual structure is then added with Equation 2.48 to produce the following:

$$
\tau^{(i)}=\bar{\beta}(q)^{-1}\left(\bar{M}(q)\left[\dot{\eta}+k_{p} e+k_{d} \dot{e}\right]+\bar{V}(q, \dot{q}) \eta\right)+\tau_{f}{ }^{(i)}
$$

where,

$$
\begin{aligned}
& \tau_{f}{ }^{(i)}=S^{(i)}\left[k_{D}{ }^{(i)} \dot{e}_{n}{ }^{(i)}+k_{S}{ }^{(i)} \dot{e}_{n}{ }^{(i)}\right] \\
& e_{n}{ }^{(i)}=\left[\begin{array}{lll}
x_{n}(t)-x_{d}(t) & y_{n}(t)-y_{d}(t) & 0
\end{array}\right]^{T}
\end{aligned}
$$

where $\tau_{f}{ }^{(i)}$ is the formation torque with $i=1,2,3$ robots, $S^{(i)^{T}} \in \mathfrak{R}^{2 \times 2}$ is matrix full rank, $k_{S}$ and $k_{D} \in \mathfrak{R}^{2 \times 2}, k_{S}$ is the virtual spring, $k_{D}$ is the virtual damper constants, $e_{n} \in \mathfrak{R}^{2 x 1}$ is the tracking error, while $k_{p}$ and $k_{d} \in \mathfrak{R}^{2 \times 2}$, $k_{p}$ and $k_{d}$ are the positive definitive diagonal matrix and $e=\eta_{d}-\eta$.

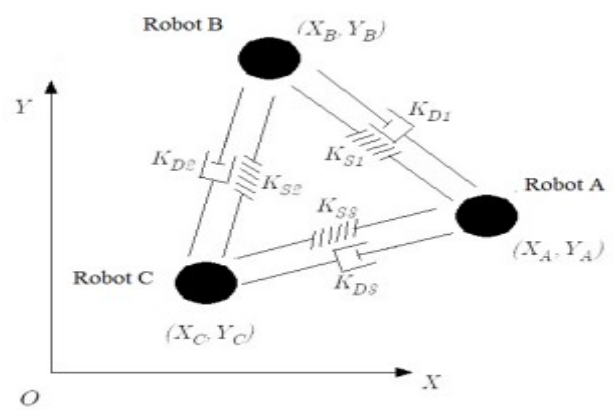

Fig. 8. The Methodology of physical concepts [4]

Figure 2 shows the formation structure, with the constituent robots connected with virtual springs and dampers. This torsion provides the correct actuation for each robot to ensure the correct formation. We use the virtual structure method to convert the entire mobile robot formation as a virtual rigid body, using the control scheme for the $i$-th robot.

From the CTC design, it is hoped that the constituent robots are able to form and maintain their designated formation when traversing their trajectory. The block diagram for the system is shown in Figure 3.

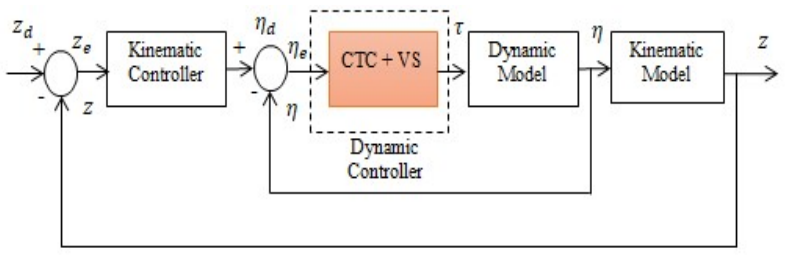

Fig. 9. Block Diagram DDMR Using CTC with Virtual Structure

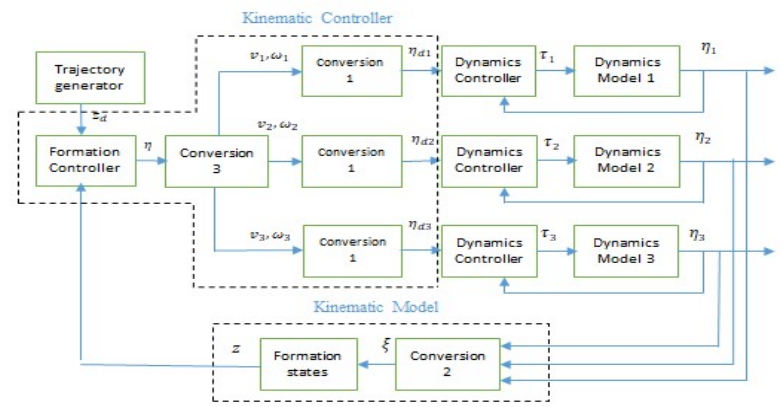

Fig. 10. Block Diagram for the formation control and trajectory tracking of mobile robotic systems

The robot kinematic and dynamic model may be designed fully by combining the control kinematics and dynamics. The robot control dynamics consists of the innerloop from the entirety of the system, where the reference is defined from the velocities of the right and left wheels. The robot control kinematics consists of the outer-loop, referencing the desired position of the robot when forming a formation.

\section{SiMULATIONS AND RESUlTS}

This section discusses the results of the design shown in Section III. We defined several tests with two trajectory shapes to test the CTC with a virtual structure, namely a circle shape and a figure-of-eight shape. Each trajectory shape will be tested with and without disturbances and noise.

\section{A. Circle Trajectory Test}

This testing stage uses the circle trajectory to prove that our designed CTC with the virtual structure can maintain formation. The robot parameters used in the test are shown in Table I.

TABLE X.
\begin{tabular}{|c|c|}
\hline Parameter & $\begin{array}{c}\text { The parameter } \\
\text { value }\end{array}$ \\
\hline$r$ & $0.025 \mathrm{~m}$ \\
$b$ & $0.1 \mathrm{~m}$ \\
$d$ & $0.05 \mathrm{~m}$ \\
$m c$ & $3 \mathrm{~kg}$ \\
$m w$ & $0.01 \mathrm{~kg}$ \\
$l c$ & $0.0327 \mathrm{~kg} \cdot \mathrm{m} 2$ \\
$l w$ & $3.1250 \mathrm{~kg} . \mathrm{m} 2$ \\
$l m$ & $1.5750 \mathrm{~kg} \cdot \mathrm{m} 2$ \\
\hline
\end{tabular}

The circle trajectory is represented in Equation (14). 
JAREE-Journal on Advance Research in Electrical Engineering Volume4, Number 1, April 2020

$\left\{\begin{array}{l}x(t)=4 \sin \left(\frac{1}{30}\right)(t) \\ y(t)=4 \cos \left(\frac{1}{30}\right)(t)\end{array}\right.$

The robot formation's movement in the $x$ and $y$ coordinates against time when following the circle trajectory is shown in Figure 5.
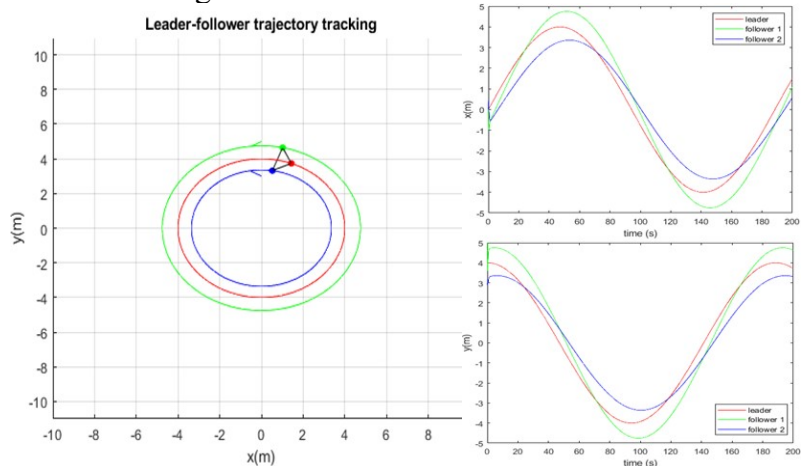

Fig. 11. Robot Formation when following a Circle Trajectory

In leader testing, the performance of our CTC design with virtual structure shows no significant error, as shown by the kinematics signal error. The kinematics signal error for this test is shown in Figures 6 to 8 .

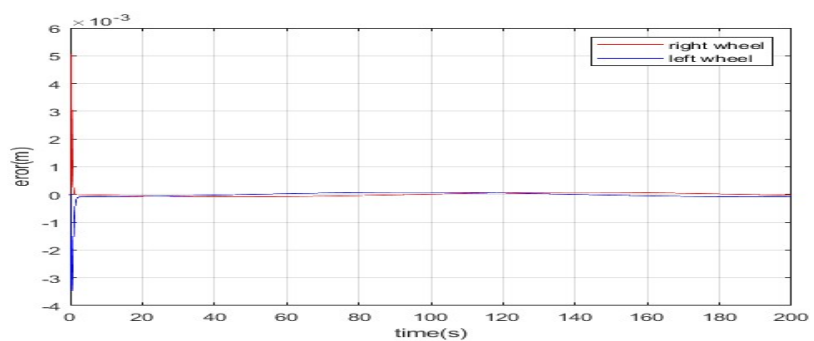

Fig. 12. Kinematics Error $\left(\mathrm{R}_{\mathrm{L}}\right)$

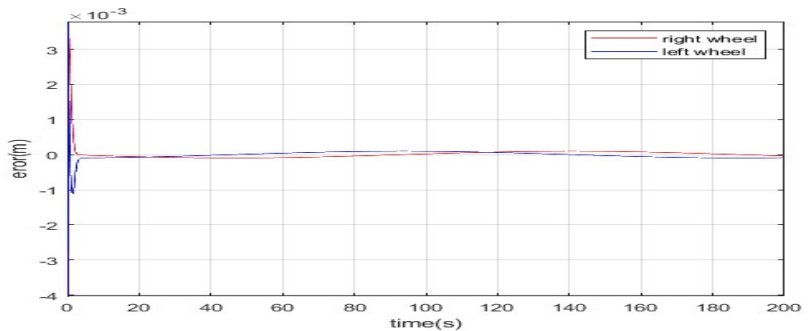

Fig. 13. Kinematics Error $\left(\mathrm{R}_{\mathrm{F} 1}\right)$

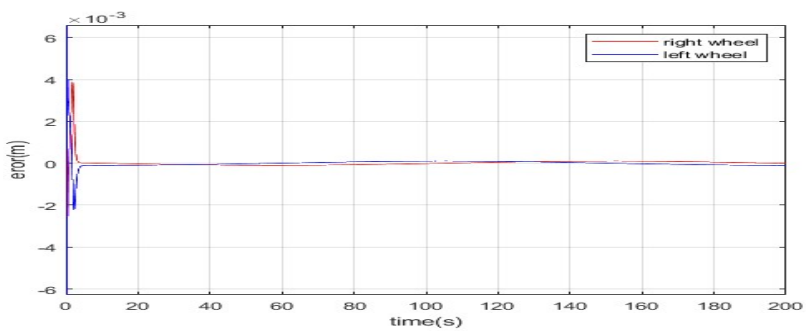

Fig. 14. Kinematics Error $\left(\mathrm{R}_{\mathrm{F} 2}\right)$
From our tests, we discover that the formation criteria have been fulfilled. Our next test is done by introducing disturbance to each robot. The applied disturbance and noise is represented by Equation (15)[9].

$$
\tau_{d}=\left(c_{c u} \operatorname{sgn}(\dot{\eta})+c_{v}(\dot{\eta})\right)
$$

where $c_{c u}=\operatorname{diag}(0.1), c_{v}=\operatorname{diag}(0.057)$ and $\dot{\eta}=\left[\begin{array}{c}\dot{\varphi}_{r} \\ \dot{\varphi}_{l}\end{array}\right]$.

The circle trajectory testing with applied disturbances is shown in Figure 9, showing that the system's response is able to follow the reference velocity signal.
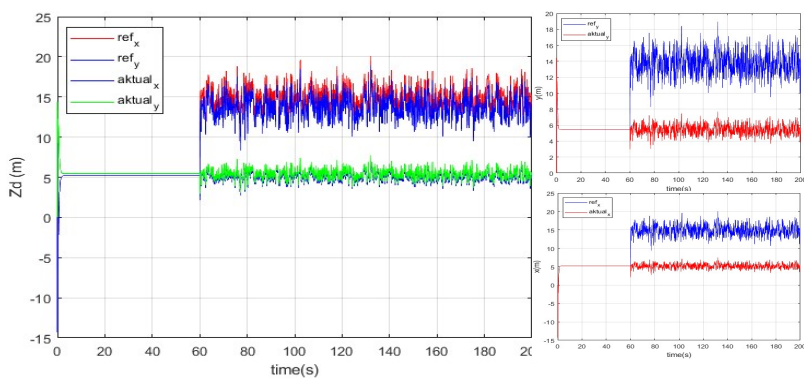

Fig. 15. Speed Reference with Disturbance and Noise

This test shows the effects of disturbances in the system, with the responses shown in Figures 10, 11 and 12. We discover that the robot formation manages to keep its shape even with the applied disturbances, shown by the MSE and error rates for each robot that is below the maximum criteria.

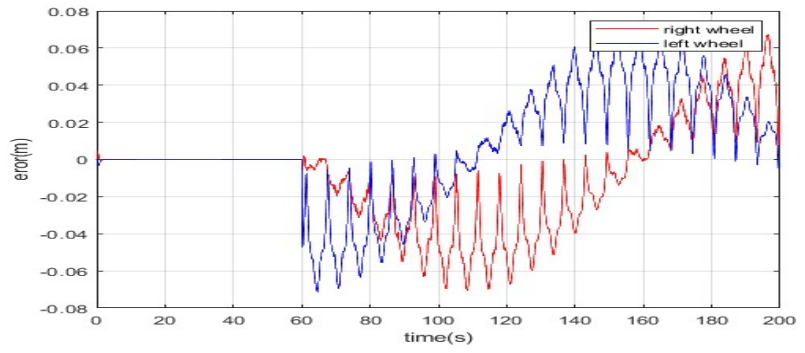

Fig. 16. Kinematics Error $\left(\mathrm{R}_{\mathrm{L}}\right)$ with Disturbance and Noise

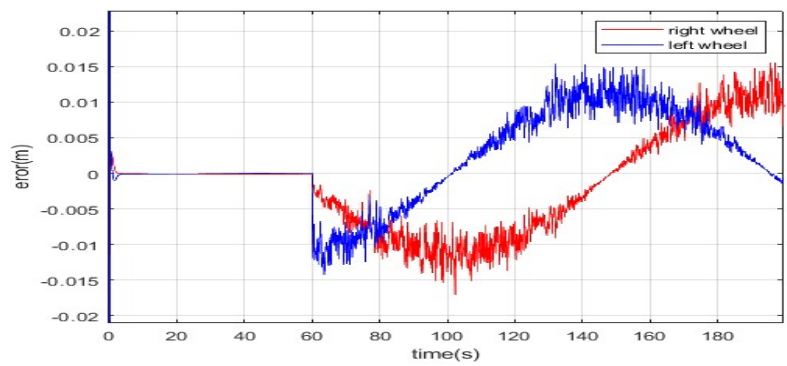

Fig. 17. Kinematics Error $\left(\mathrm{R}_{\mathrm{F} 2}\right)$ with Disturbance and Noise 
JAREE-Journal on Advance Research in Electrical Engineering Volume4, Number 1, April 2020

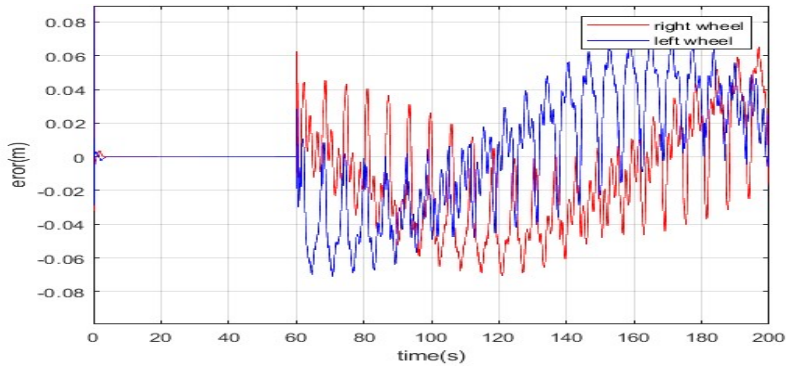

Fig. 18. Kinematics Error $\left(\mathrm{R}_{\mathrm{F} 1}\right)$ with Disturbance and Noise

\section{B. Figure-of-Eight Trajectory Testing}

The second test uses the figure-of-eight reference trajectory, using the trajectory equation shown in Equation (16). The robot formation's movement in the $x$ and $y$ coordinates against time when following the figure-of-eight trajectory is shown in Figure 13.
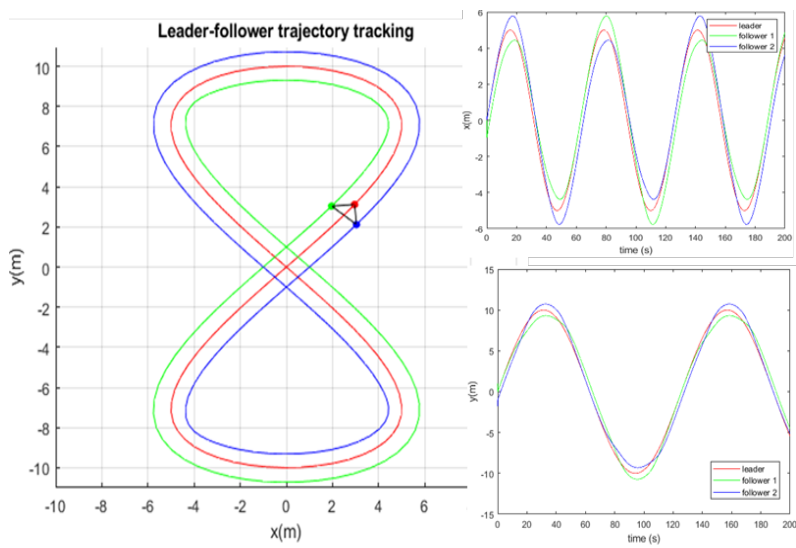

Fig. 19. Robot Formation when following Figure-of-Eight Trajectory

The eight-shape trajectory is represented in Equation (16).

$$
\left\{\begin{array}{l}
x(t)=5 \sin \left(\frac{1}{10}\right)(t) \\
y(t)=10 \cos \left(\frac{1}{20}\right)(t)
\end{array}\right.
$$

The test results are in the form of MSE values in the tracking error signal in the kinematics of each robot. The obtained error signals show that the system can maintain formation and follow the given trajectory.

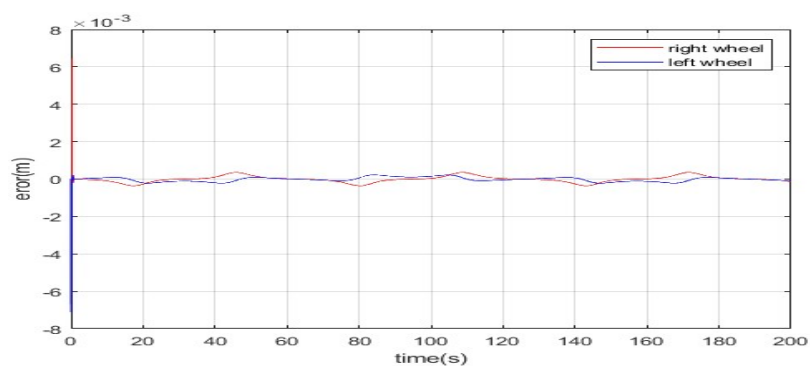

Fig. 20. Kinematic Error $\left(\mathrm{R}_{\mathrm{L}}\right)$

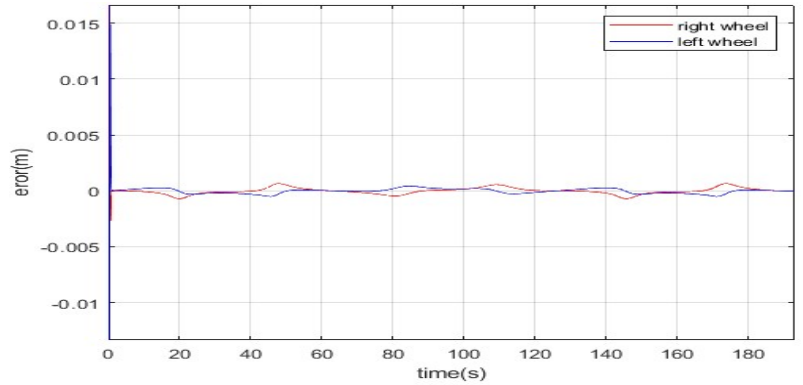

Fig. 21. Kinematic Error $\left(\mathrm{R}_{\mathrm{F} 1}\right)$

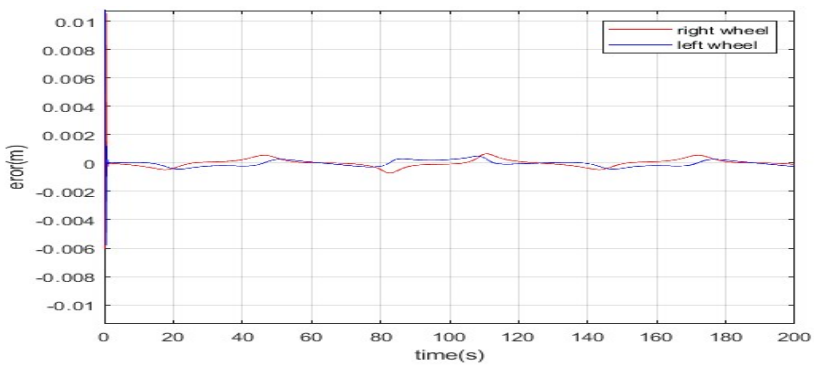

Fig. 22. Kinematic Error $\left(\mathrm{R}_{\mathrm{F} 2}\right)$

The next test is disturbance testing. This test is fundamentally similar to the disturbance test on the circle trajectory test suite before. Figures 17, 18 and 19 show the disturbance effects to the formation as shown by the MSE values for each robot.

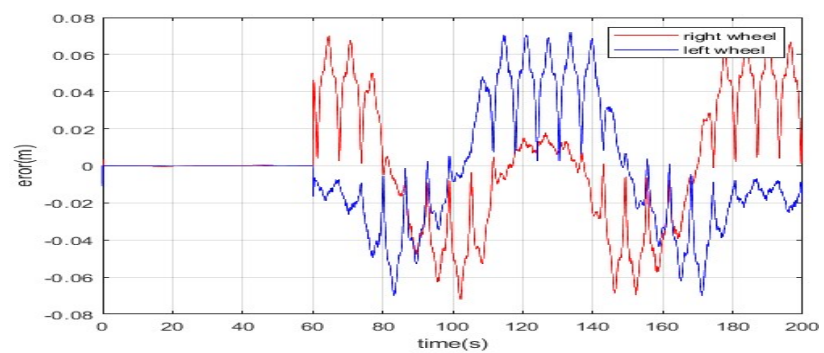

Fig. 23. Kinematic Error $\left(\mathrm{R}_{\mathrm{L}}\right)$ with Disturbance and Noise

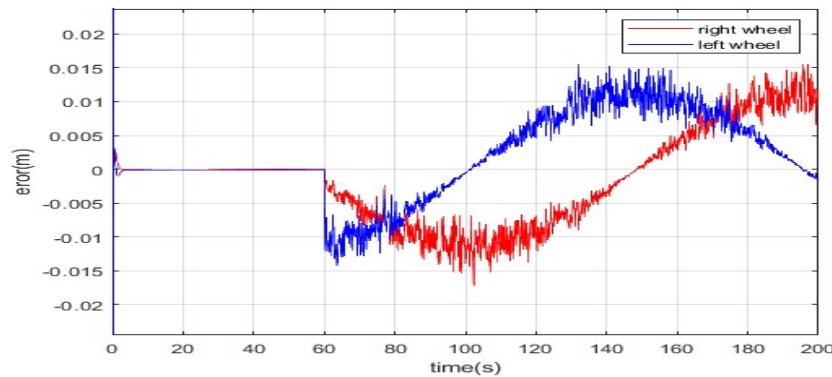

Fig. 24. Kinematic Error $\left(\mathrm{R}_{\mathrm{F} 1}\right)$ with Disturbance and Noise 
JAREE-Journal on Advance Research in Electrical Engineering Volume4, Number 1, April 2020

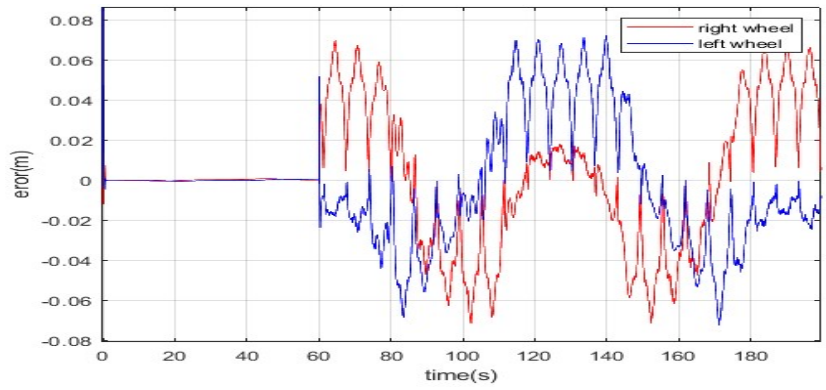

Fig. 25. Kinematic Error $\left(\mathrm{R}_{\mathrm{F} 1}\right)$ with Disturbance and Noise

\section{CONCLUSIONS}

From our tests, we conclude that the tracking problems of multi-robot formations in a given trajectory while holding a triangular formation can be solved by applying a virtual structure controller and a linear algebra approach. From the non-disturbance tests of both circle and eight-shape tracks, the system can hold formation while following its given trajectory, showing the best error rates among the tests with $\mathrm{MSE}_{x}=4.5152 \times 10^{-10}$ and $\mathrm{MSE} e_{y}=3.3945 \times 10^{-10}$. Disturbance testing on the two tracks shows that the system is able to cope with outside disturbance, as shown in the eight-shape test where even with a relatively high MSE increase in follower 2 with $\mathrm{MSE}_{x}=0.000010$ and $\mathrm{MSE}_{y}$ $=0.000011$, the formation is able to hold while traversing the track with error rates within the set criteria.

Thus, it may be concluded that the proposed control system by using the virtual structure and the linear algebra approach can control and maintain a triangular formation tracking a given trajectory with or without disturbance.

\section{REFERENCES}

[1] J. Alonso-Mora, S. Baker, and Daniela Rus, "Multi-robot formation control and object transport in dynamic environments via constrained optimization", international Journal of Robotics Research. 2017

[2] T. Okimoto, Tony Ribeiro, B, Damien, and I. Katsumi, "Mission Oriented Robust Multi-Team Formation and Its Application to Robot Rescue Simulation" International Joint Conference on Artificial Intelligence (IJCAI), 2016

[3] M. Sahal, T. Agustinah, and A. Jazidie, "Switching Formation and Topology in Cooperative Multi-Agent Source Seeking Using Gradient Estimation"International Conference of Artificial Intelligence and Information Technology (ICAIIT), pp. 151-156, 2019

[4] A. Kusuma and T. Agustinah, "Disturbance Compensation Using CTC with NDOB for Formation Control of Mobile Robots", International Conference on Information and Communications Technology (ICOIACT), pp. 673-678, 2018.

[5] A. Adel, S. Ali A. Moosavian, A. Khalil and K. N. Toosi " $A$ Virtual Structure-based Approach to Formation Control of Cooperative Wheeled Mobile Robots", International Conference on Robotics and Mechatronics February 13-15, 2013.

[6] R. Andres, S. Gustavo, Vicente Mut and Fernando di Sciascio, "Formation control and trajectory tracking of mobile robotic systems - a Linear Algebra approach", Cambridge University Press 2010. Robotica volume 29, pp. 335-349, 2011.

[7] D. Rached, H. A. Ahmad "Dynamic Modelling of DifferentialDrive Mobile Robots using Lagrange and Newton-Euler Methodologies", A Unified Framework," Adv. Robot. Autom. 2013, vol. 02 , no. 02.2013

[8] F. L. Lewis, C. T. Abdallah, D. M. Dawson, and F. L. Lewis,
Robot manipulator control: theory and practice, 2nd ed., And expanded. New York: Marcel Dekker, 2004.

[9] D. Phaoharuhansa and A. Shimada, "Friction Compensation for Two Wheeled Iinverted Pendulum Robot Using System Identification," Int. J. Adv. Sci. Technol. 